\title{
Dynamics of Transversely Vibrating Pipes under Non-classical Boundary Conditions
}

\author{
Begüm Y. Dağlı ${ }^{*}$, B. Gültekin Sınır \\ Department of Civil Engineering, Celal Bayar University, Turkey
}

Copyright $@ 2015$ Horizon Research Publishing All rights reserved.

\begin{abstract}
The transverse of free vibration of pipes conveying fluid has been examined by using Euler-Bernoulli beam theory to show the effect of varied boundary conditions on pipes dynamic behaviors. The equation of motion of the pipe conveying fluid is obtained with a new approach with the assumptions of ideal fluid, which moves in the vertical direction with pipe and the pipe makes small oscillations, by Hamilton's variation principle. The flow in the pipe was modeled by considering well-known Euler equation. The dimensionless equations are solved for two different set of non-classical boundary conditions. The natural frequency equations and the critical flow velocity equations are obtained and the relation between the mass ratio and vibration frequency is examined by solving the differential equations. The values of natural frequencies caused by the fluid velocity are presented graphically.
\end{abstract}

Keywords Pipes Conveying Fluid, Natural Frequency, Non-classical Boundary Conditions

\section{Introduction}

The dynamic behavior and stability of pipes conveying fluid has been investigated thoroughly by many researchers due to technological importance. As early as 1878 a series of experiments have been done on travelling chains and elastic cords by Aitken. Then Brillouin studied on cantilevered pipe conveying fluid (1885). Benjamin (1961) was the first to provide a comprehensive study on pipe vibrations. Housner (1952) was the first to derive the correct governing equation of motion of a pipe conveying fluid. There are many studies on pipe dynamic especially modeled as Euler Bernoulli beam in the literature. By using the Euler Bernoulli theory following studies are done: nonlinear vibration of the pipe Paidoussis (1988), analysis of vibration and stability of slightly curved pipes Sinir (2010), full curved pipes Chen (1972-1973) etc. There are also studies which used different beam theories. For example, Ergut et al.(2011) examined the pipe dynamic behavior using Rayleigh beam theory.
The linear equations of motion using Timoshenko beam theory was improved and non-dimensionalized by Paidoussis (1976)- and Laithier (1981) taking plug-flow into account for the first time.

In the present paper, dynamical behavior of pipes which modeled by using Euler-Bernoulli theory are investigated by considering two set of non-classical boundary conditions.

The equations of motion of beam have been achieved with the variational approach, which accounts for the exchange of energy between a flowing fluid and a pipe. (Han et al.,1999) Then the equations have been rendered dimensionless to obtain universal results. Non-dimensional velocity $u$, stiffnesses of translational springs $k_{l}, k_{3}$; stifnesses of rotational springs $k_{2}, k_{4}$, damping coefficient $\mu$ and also mass ratio $\beta$ is obtained for Euler-Bernoulli pipe model. Solutions of linear differential equations have been studied by analytical methods by applying two set of non-classical boundary conditions. The non-classical boundary conditions take into account the shape deflection curve and the additional mass, the damper, as well as the translational and rotational springs at the boundaries. As a result of the analysis the effect of stifnesses of translation-rotational springs and damping coefficient on the fluid velocity and natural frequency is given in figures for each boundary condition. Besides, some critical velocities for the pipe instability have been obtained by considering Euler Theory.

\section{Assumptions and Equation of Motion}

The system which is studied in the present paper consists of a pipe of length $L$, cross-sectional area $A_{p}$, mass per unit length $m$, the modulus of elasticity $E$, density $\rho_{p}$ and area moment of inertia $I$. The fluids terms are represented as shown; mass $M$, per unit length with axial flow velocity $u$ and density of the fluid as $\rho_{f}$. The fluid is incompressible and inviscid, pipe is composed by uniform and homogeneous material. System's motion is planar. Under these assumptions the Lagrangian for Euler Bernoulli beam can be written as 


$$
\begin{aligned}
& \mathcal{L}=\frac{1}{2} \int_{0}^{L} \rho_{p} A_{p}\left(\frac{\partial v^{*}\left(x^{*}, t^{*}\right)}{\partial t^{*}}\right)^{2} d x^{*} \\
& -\frac{1}{2} \int_{0}^{L} E I\left(\frac{\partial^{2} v^{*}\left(x^{*}, t^{*}\right)}{\partial x^{* 2}}\right) d x^{*}+\delta W_{n c}
\end{aligned}
$$

The first term in equation (1) is the kinetic energies and the following term is the potential energies of the beam. Where, $v^{*}\left(x^{*}, t^{*}\right)$ is the transverse deflection at the axial location $x^{*}$ and time $t^{*}, \delta W_{n c}$ is virtual work done by non-conservative forces (which involves structural damping and forces) [2]. In this study, the virtual work consists of only damping of pipe and the damping is assumed to follow the Kelvin-Voigt model. The work done by non-conservative forces can be expressed as

$$
\delta W_{n c}=-\int_{0}^{L} \eta I \dot{v}^{\prime \prime} \delta v^{\prime \prime} d x
$$

Where $\eta$ is the damping coefficient.

Using the Hamilton's principle for single dependent and two independent variables,

$$
\delta \int_{t_{1}}^{t_{2}} \mathcal{L} d t
$$

the well-known governing equation for Euler Bernoulli model is obtained as follows;

$$
\rho_{p} A_{p} \ddot{v}^{*}+E I v^{* l v}+\eta I \dot{v}^{* I V}=0
$$

Representation of the derivatives with respect to the spatial variable is shown by ()$^{\prime}$ and the derivatives with respect to time shown by $\left({ }^{\cdot}\right)$. Superscript * symbols are used to indicate the dimensional quantities.

To derive the equation of motion of Euler-Bernoulli pipe, flow in lateral direction is investigated by using Euler equation. The equation for ideal fluids can be written as;

$$
\frac{\partial P}{\partial y^{*}}=\rho_{f} Y-\rho_{f} a_{y}
$$

Where, $\mathrm{y}$ is the vertical deflection of the pipe, $P$ is the pressure, vertical components internal force is $Y$, and its acceleration of fluid in motion is $a_{y}$. The fluid and the pipe move together in vertical direction. Thus, the equation of motion of the Euler- Bernoulli pipe conveying fluid will be obtained by superposing the acceleration terms of the beam with the fluid term. The differential equation of a pipe can be expressed as

$$
\rho_{p} A_{p} \ddot{v}^{*}+\rho_{f} A_{f}\left(\ddot{v}^{*}+2 u^{*} \dot{v}^{\prime}+u^{* 2} v^{* \prime}\right)+E I v^{* I V}+\eta I \dot{v}^{* I V}=0
$$

The equation is made dimensionless though the definitions

$$
\begin{gathered}
v=\frac{v^{*}}{r} \quad x=\frac{x^{*}}{L} \quad t=t^{*} \sqrt{\frac{E I}{L^{4}(m+M)}} \\
\eta=\eta^{*} \frac{I}{L^{2} \sqrt{E I(m+M)}} \quad u=u^{*} \frac{\sqrt{M}}{\sqrt{E I}} L
\end{gathered}
$$

Here, $r$ indicates the radius of gyration which is used to non-dimensionalize the displacement. Introducing dimensionless parameters, Eq. (6) becomes:

$$
v^{l v}+u^{2} v^{\prime \prime}+2 \sqrt{\beta} u \dot{v}^{\prime}+\ddot{v}+\eta \dot{v}^{l v}=0
$$

Where $\beta$ is the relative mass of fluid and can be written as

$$
\beta=\frac{M}{(m+M)}
$$

\section{Analytical Solutions}

In this section, we search for exact solution of homogeneous, linear ordinary differential equations with the associated boundary conditions. The dynamic behavior of the pipe can be assumed to be harmonic because the equations of motion is linear. In this case, the general solution can be given as

$$
v(x, t)=X_{n}(x) e^{i \omega_{n} t}+\bar{X}_{n}(x) e^{-i \omega_{n} t}
$$

Where, $X_{n}$ represents a complex function because of the Coriolis term and $\omega_{n}$. is the natural frequency.

The substitution of equation (10) into the dimensionless equation of motion gives

$$
\left(1+i \omega_{n} \eta\right) X_{n}{ }^{v}+u^{2} X_{n}^{\prime \prime}+2 \sqrt{\beta} u i \omega_{n} X_{n}^{\prime}-\omega_{n}^{2} X_{n}=0
$$

The general solution of the homogeneous differential equations with the arbitrary constants has the form

$$
X(x)=\sum_{j=1}^{4} C_{j} e^{m_{j} x}
$$

In this expression, $m_{j}$ and $C_{j}$ are unknown terms. The equation,

$$
a_{4} m^{4}+a_{3} m^{3}+a_{2} m^{2}+a_{1} m+a_{0}=0
$$

is called the characteristic equation and $a_{i}$ coefficients have been described for Euler-Bernoulli theory as shown below; transactions have been made suitable for the computer program.

$$
\begin{gathered}
a_{0}=-\omega_{n}^{2} \quad a_{1}=+2 \sqrt{\beta} u i . \omega_{n} \quad a_{2}=+u^{2} a_{3}=0 \\
a_{4}=1+i \omega_{n} \eta
\end{gathered}
$$

\subsection{Non-classical Boundary Conditions}

In this study two different cases of support at the ends of 
the pipe are investigated, as shown in Table 1.

The boundary conditions pairs are expressed as

$$
\begin{array}{ll}
\text { at } \mathrm{x}=0 & v \rightarrow \eta I \dot{v}^{\prime \prime \prime}+E I v^{\prime \prime \prime}=-M u\left(\dot{v}+u v^{\prime}\right) \\
& v^{\prime} \rightarrow \eta I \dot{v}^{\prime \prime}+E I v^{\prime \prime}=0 \\
\text { at } \mathrm{x}=\mathrm{L} & v \rightarrow \eta I \dot{v}^{\prime \prime \prime}+E I v^{\prime \prime \prime}=0 \\
& v^{\prime} \rightarrow \eta \dot{v}^{\prime \prime}+E I v^{\prime \prime}=0
\end{array}
$$

The shear natural boundary condition at $\mathrm{x}=0$ is different than at $\mathrm{x}=\mathrm{L}$ because of the flow out-release effect. [2]

Parameters $k_{1}, k_{3}$ are stiffnesses of translational springs; $k_{2}$, $k_{4}$ are the stifnesses of rotational springs.

In the first case, pipe conveying fluid is considered with transversal and rotational springs at the left and right-hand ends. At that rate the dimensionless boundary conditions can be given as

$$
\begin{aligned}
x=0 & \rightarrow \eta \dot{v}^{\prime \prime \prime}+v^{\prime \prime \prime}+\sqrt{\beta} u \dot{v}+u^{2} v^{\prime}-k_{1} v=0 \\
& \rightarrow v^{\prime \prime}+\eta \dot{v}^{\prime \prime}-k_{2} v^{\prime}=0 \\
x= & \rightarrow \eta \dot{v}^{\prime \prime \prime}+v^{\prime \prime \prime}+k_{3} v=0 \\
& \rightarrow v^{\prime \prime}+\eta \dot{v}^{\prime \prime}+k_{4} v^{\prime}=0
\end{aligned}
$$

In the second case, pipe with transversal and rotational springs at the left- hand and elastic clamped with a concentrated mass at the right-hand end, is investigated. The dimensionless boundary conditions corresponding to Case II. Become

$$
\begin{aligned}
x=0 & \rightarrow v=0 \\
& \rightarrow \eta \dot{v}^{\prime \prime}+v^{\prime \prime}-k_{2} v^{\prime}=0 \\
x=L & \rightarrow \eta \dot{v}^{\prime \prime \prime}+v^{\prime \prime \prime}=0 \\
& \rightarrow \eta \dot{v}^{\prime \prime}+v^{\prime \prime}+k_{4} v^{\prime}=0
\end{aligned}
$$

Where the dimensionless parameters are

$$
\begin{array}{ll}
k_{1}=k_{1}^{*} \frac{L^{3}}{E I}, & k_{2}=k_{2}^{*} \frac{L}{E I} \\
k_{3}=k_{3}^{*} \frac{L^{3}}{E I}, & k_{4}=k_{4}^{*} \frac{L}{E I}
\end{array}
$$

\begin{tabular}{|c|c|}
\hline Boundary Conditions & At left end $(x=0)$ \\
\hline \multirow{2}{*}{$\begin{array}{c}\text { Case I. } \\
\text { Elastic Clamped end }\end{array}$} & At right end $(\mathrm{x}=\mathrm{L})$ \\
\hline & At left end \\
\hline \multirow{2}{*}{$\begin{array}{l}\text { Case II. } \\
\text { Pinned end with torsional spring \& sliding end with } \\
\text { torsional spring }\end{array}$} & At right end $(x=L)$ \\
\hline & $k_{4}^{k^{\prime}}$ \\
\hline
\end{tabular}

As a result of the substitution the boundary conditions into the dimensionless equation of motion, four algebraic equations are obtained to determine the arbitrary constants. The equations which gives the arbitrary constants can be represented by a matrix form as

\section{Case I.}

$$
\left[\begin{array}{cccc}
(\eta i \omega+1) m_{1}^{3}+u^{2} m_{1}+\sqrt{\beta} u i \omega-k_{1} & (\eta i \omega+1) m_{1}^{3}+u^{2} m_{1}+\sqrt{\beta} u i \omega-k_{1} & (\eta i \omega+1) m_{1}^{3}+u^{2} m_{1}+\sqrt{\beta} u i \omega-k_{1} & (\eta i \omega+1) m_{1}^{3}+u^{2} m_{1}+\sqrt{\beta} u i \omega-k_{1} \\
(\eta i \omega+1) m_{1}^{2}-k_{3} m_{1} & (\eta i \omega+1) m_{2}^{2}-k_{3} m_{2} & (\eta i \omega+1) m_{3}^{2}-k_{3} m_{3} & (\eta i \omega+1) m_{4}^{2}-k_{3} m_{4} \\
(\eta i \omega+1) m_{1}^{3} e^{m_{1}}+k_{3} e^{m_{1}} & (\eta i \omega+1) m_{1}^{3} e^{m_{2}}+k_{3} e^{m_{2}} & (\eta i \omega+1) m_{1}^{3} e^{m_{3}}+k_{3} e^{m_{3}} & (\eta i \omega+1) m_{1}^{3} e^{m_{4}}+k_{3} e^{m_{4}} \\
(\eta i \omega+1) m_{1}^{2} e^{m_{1}}+k_{4} m_{1} e^{i m_{1}} & (\eta i \omega+1) m_{2}^{2} e^{m_{2}}+k_{4} m_{2} e^{i m_{2}} & (\eta i \omega+1) m_{3}^{2} e^{m_{1}}+k_{4} m_{3} e^{i m_{3}} & (\eta i \omega+1) m_{4}^{2} e^{m_{1}}+k_{4} m_{4} e^{i m_{4}}
\end{array}\right]\left[\begin{array}{l}
C_{1} \\
C_{2} \\
C_{3} \\
C_{4}
\end{array}\right]=\left[\begin{array}{l}
0 \\
0 \\
0 \\
0
\end{array}\right]
$$

Case II.

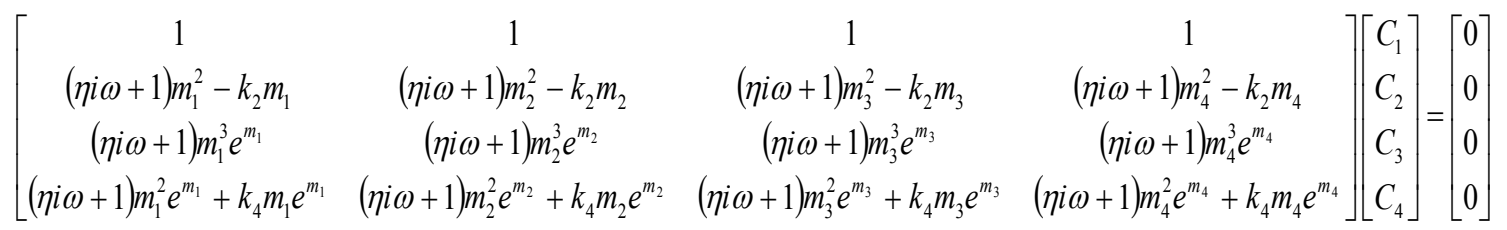

Table 1. Non-classical bounday conditions 
The determinant of the matrix of coefficients must be equal to zero to obtain a non-trivial solution. In this case, the problem becomes the well-known eigenvalue-eigenvector problem. The eigenvalues represent natural frequencies and the eigenvectors are corresponded to the mode shapes.

\section{Numerical Results}

Numerical values of the natural frequencies are obtained for the initial three modes in this section. In Table 2. the first three frequencies are presented for elastic clamped- elastic clamped boundary condition (Case I) for various stiffnesses of translational and rotational springs. To verify the effect of fluid velocity on the natural frequencies five values of $u=0.10,0.50,1.00,1.50$ and 2.00 are assumed.

Table 2. Non-classical bounday conditions $\beta=0.2, \eta=0.01$

\begin{tabular}{|c|c|c|c|c|c|c|c|c|}
\hline $\mathrm{u}$ & $\mathrm{k}_{1}$ & $\omega_{1}$ & $\omega_{2}$ & $\omega_{3}$ & $\mathrm{k}_{2}$ & $\omega_{1}$ & $\omega_{2}$ & $\omega_{3}$ \\
\hline \multirow{4}{*}{0.10} & 0.00 & 4.0804 & 25.2907 & 62.3429 & 0.00 & 1.6827 & 23.6354 & 60.4502 \\
\hline & 10.00 & 1.9274 & 24.5766 & 62.0327 & 10.00 & 5.8557 & 29.4037 & 68.9634 \\
\hline & 100.00 & 1.4857 & 20.6727 & 59.5307 & 100.00 & 6.4416 & 31.2083 & 71.0620 \\
\hline & 1000.00 & 1.4471 & 18.0014 & 52.7894 & 1000.00 & 6.5109 & 31.4355 & 71.1243 \\
\hline \multirow{4}{*}{0.50} & 0.00 & 3.8967 & 25.1142 & 62.0935 & 0.00 & 1.0363 & 23.4416 & 60.1920 \\
\hline & 10.00 & 1.7829 & 24.3973 & 61.7828 & 10.00 & 5.7531 & 29.2561 & 68.7329 \\
\hline & 100.00 & 1.3977 & 20.4966 & 59.2763 & 100.00 & 6.3472 & 31.0593 & 70.8149 \\
\hline & 1000.00 & 1.3639 & 17.8759 & 52.5594 & 1000.00 & 6.4170 & 31.2854 & 70.8743 \\
\hline \multirow{4}{*}{1.00} & 0.00 & 3.2794 & 24.6321 & 61.5267 & 0.00 & - & 22.9065 & 59.6020 \\
\hline & 10.00 & 1.3130 & 23.9064 & 61.2146 & 10.00 & 5.4349 & 28.8733 & 68.2283 \\
\hline & 100.00 & 1.0873 & 20.0310 & 58.6978 & 100.00 & 6.0638 & 30.6923 & 70.3046 \\
\hline & 1000.00 & 1.0670 & 17.5401 & 52.0584 & 1000.00 & 6.1367 & 30.9189 & 70.3624 \\
\hline \multirow{4}{*}{1.50} & 0.00 & 1.9226 & 23.8431 & 60.6697 & 0.00 & - & 22.0257 & 58.7075 \\
\hline & 10.00 & 0.0001 & 23.1026 & 60.3552 & 10.00 & 4.8704 & 28.2621 & 67.4782 \\
\hline & 100.00 & - & 19.2835 & 57.8228 & 100.00 & 5.5734 & 30.1181 & 69.5659 \\
\hline & 1000.00 & - & 16.9973 & 51.3160 & 1000.00 & 5.6527 & 30.3474 & 69.6243 \\
\hline \multirow{4}{*}{2.00} & 0.00 & 1.6189 & 22.7166 & 59.5111 & 0.00 & - & 20.7603 & 57.4955 \\
\hline & 10.00 & - & 21.9551 & 59.1931 & 10.00 & 3.9705 & 27.4085 & 66.4745 \\
\hline & 100.00 & - & 18.2383 & 56.6396 & 100.00 & 4.8219 & 29.3260 & 68.5919 \\
\hline & 1000.00 & - & 16.2321 & 50.3256 & 1000.00 & 4.9133 & 29.5603 & 68.6532 \\
\hline $\mathrm{u}$ & $\mathrm{k}_{3}$ & $\omega_{1}$ & $\omega_{2}$ & $\omega_{3}$ & $\mathrm{k}_{4}$ & $\omega_{1}$ & $\omega_{2}$ & $\omega_{3}$ \\
\hline \multirow{4}{*}{0.10} & 0.00 & 4.0734 & 25.2907 & 62.3430 & 0.00 & 1.7038 & 23.6366 & 60.4512 \\
\hline & 10.00 & 1.9224 & 24.5758 & 62.0320 & 10.00 & 5.8526 & 29.4021 & 68.9585 \\
\hline & 100.00 & 1.4792 & 20.6480 & 59.5259 & 100.00 & 6.4388 & 31.2118 & 71.0746 \\
\hline & 1000.00 & 1.4403 & 18.0065 & 52.8115 & 1000.00 & 6.5082 & 31.4401 & 71.1405 \\
\hline \multirow{4}{*}{0.50} & 0.00 & 3.9106 & 25.1149 & 61.0939 & 0.00 & 1.2999 & 23.4598 & 60.2014 \\
\hline & 10.00 & 1.6312 & 24.3904 & 61.7790 & 10.00 & 5.6613 & 29.2122 & 68.6852 \\
\hline & 100.00 & 1.2058 & 20.4809 & 59.2466 & 100.00 & 6.2426 & 31.0272 & 70.8407 \\
\hline & 1000.00 & 1.1685 & 17.8453 & 52.6387 & 1000.00 & 6.3113 & 31.2573 & 70.9173 \\
\hline \multirow{4}{*}{1.00} & 0.00 & 3.3632 & 24.6345 & 61.5277 & 0.00 & - & 22.9749 & 59.6316 \\
\hline & 10.00 & 0.0134 & 23.8837 & 61.2057 & 10.00 & 5.0259 & 28.6940 & 68.0744 \\
\hline & 100.00 & - & 19.9160 & 58.6235 & 100.00 & 5.5935 & 30.5014 & 70.2632 \\
\hline & 1000.00 & - & 17.3336 & 52.1385 & 1000.00 & 5.6604 & 30.7319 & 70.3524 \\
\hline \multirow{4}{*}{1.50} & 0.00 & 2.2821 & 23.8486 & 60.6715 & 0.00 & - & 22.1793 & 58.7687 \\
\hline & 10.00 & - & 23.0513 & 60.3394 & 10.00 & 3.7333 & 27.8484 & 67.1577 \\
\hline & 100.00 & - & 18.9643 & 57.6864 & 100.00 & 4.3038 & 29.6319 & 69.3622 \\
\hline & 1000.00 & - & 16.4483 & 51.3151 & 1000.00 & 4.3696 & 29.8607 & 69.4629 \\
\hline \multirow{4}{*}{2.00} & 0.00 & 1.1356 & 22.7272 & 59.5139 & 0.00 & - & 21.0404 & 57.6009 \\
\hline & 10.00 & - & 21.8546 & 59.1684 & 10.00 & - & 26.6457 & 65.9237 \\
\hline & 100.00 & - & 17.5623 & 56.4197 & 100.00 & - & 28.3886 & 68.1262 \\
\hline & 1000.00 & - & 15.1279 & 50.1547 & 1000.00 & 1.1590 & 28.6134 & 68.2374 \\
\hline
\end{tabular}


As shown in Table 2, while $k_{l}$ increases and other parameters keep constant $\left(\mathrm{k}_{2}, \mathrm{k}_{3}, \mathrm{k}_{4}=1\right)$, the values of the natural frequencies for the first, second and third mode are lower. When $u=1.50$, the numerical results cannot be calculated for the values 100 and 1000 of $k_{l}$ and also natural frequencies cannot be obtained for $k_{l}=10$ assuming the fluid velocity as 2 at first mode. Due to the different $k_{3}$, the values of natural frequencies are similar to the values obtained with various $k_{l}$. On the other hand, natural frequencies increase with increase in value of the stiffnesses of rotational springs $\mathrm{k}_{2}, \mathrm{k}_{4}$

Table 3 shows the correlation between the values of natural frequencies and stiffnesses $\left(k_{2}, k_{4}\right)$ for pinned end with torsional spring - sliding end with torsional spring. As the support translational stiffness increases, natural frequencies increase. Stiffness parameter gives less change on the natural frequencies between the values of 100 and 1000. According to Table 3, it is verified that stiffness of rotational spring slightly changes natural frequencies. When $k_{4}$ increases, values of the first, second and third dimensionless natural frequencies increase too. . The result cannot be taken at the first mode for $k_{2}=0.00$ assuming the fluid velocity as 1.50 and 2.00 , for $k_{4}=10.00,100.00$,
1000.00 in case of $u=2.00$.

The pipes conveying fluid under nonclassical boundary conditions behave as pipes with classical supports for special values of stiffnesses of translational and rotational springs. In this section variation of natural frequencies investigated for four special cases. For the analysis, $\mathrm{k}_{1}, \mathrm{k}_{2}, \mathrm{k}_{3}, \mathrm{k}_{4}$ are assumed as $0, \infty, \infty, 0$ respectively for Case I. Under these values of coefficients, Case I corresponded to guided-pinned pipe. For free-clamped condition, $\mathrm{k}_{1}, \mathrm{k}_{2}, \mathrm{k}_{3}, \mathrm{k}_{4}$ were used as 0 , $0, \infty, \infty$ for Case İI. In Case III, $\mathrm{k}_{1}, \mathrm{k}_{2}, \mathrm{k}_{3}, \mathrm{k}_{4}$ were considered as $\infty, \infty, 0, \infty$ to correspond clamped-guided condition. The last one was designed as clamped-pinned condition assuming $\mathrm{k}_{1}, \mathrm{k}_{2}, \mathrm{k}_{3}, \mathrm{k}_{4}$ as $\infty, \infty$,, 0 for Case IV. As shown in Fig. 1, the values of natural frequencies decrease with increasing fluid velocity. Due to the different pipe ends conditions, the natural frequencies are very different to each other. For Case I and II, the stifnesses of the supports give no visible change to the critical velocity. For Case IV, the natural frequency and critical velocity approach maximum values. According to the results the natural frequencies are higher for a pipe under tensile effects due to the immovable boundary conditions.

\begin{tabular}{|c|c|c|c|c|c|c|c|c|}
\hline $\mathrm{u}$ & $\mathrm{k}_{2}$ & $\omega_{1}$ & $\omega_{2}$ & $\omega_{3}$ & $\mathrm{k}_{4}$ & $\omega_{1}$ & $\omega_{2}$ & $\omega_{3}$ \\
\hline \multirow{4}{*}{0.10} & 0.00 & 1.4155 & 16.9254 & 50.1793 & 0.00 & 1.5563 & 16.2004 & 49.3388 \\
\hline & 10.00 & 3.6106 & 20.9099 & 56.0241 & 10.00 & 2.9777 & 21.3322 & 57.1109 \\
\hline & 100.00 & 4.1388 & 23.1840 & 60.1037 & 100.00 & 3.1690 & 22.7646 & 59.4501 \\
\hline & 1000.00 & 4.2079 & 23.5402 & 60.5189 & 1000.00 & 3.1912 & 22.9462 & 59.6483 \\
\hline \multirow{4}{*}{0.50} & 0.00 & 1.3106 & 16.8052 & 49.9831 & 0.00 & 1.5329 & 16.0896 & 49.1467 \\
\hline & 10.00 & 3.5756 & 20.8055 & 55.8408 & 10.00 & 2.8808 & 21.1979 & 56.8978 \\
\hline & 100.00 & 4.1041 & 23.0729 & 59.9017 & 100.00 & 3.0633 & 22.6300 & 59.2656 \\
\hline & 1000.00 & 4.1729 & 23.4264 & 60.3106 & 1000.00 & 3.0845 & 22.8122 & 59.4714 \\
\hline \multirow{4}{*}{1.00} & 0.00 & 0.9092 & 16.4796 & 49.5437 & 0.00 & 1.4580 & 15.7904 & 48.7157 \\
\hline & 10.00 & 3.4710 & 20.5290 & 55.4342 & 10.00 & 2.5598 & 20.8303 & 56.4240 \\
\hline & 100.00 & 4.0060 & 22.7942 & 59.4761 & 100.00 & 2.7121 & 22.2461 & 58.8128 \\
\hline & 1000.00 & 4.0747 & 23.1447 & 59.8778 & 1000.00 & 2.7298 & 22.4270 & 59.0269 \\
\hline \multirow{4}{*}{1.50} & 0.00 & - & 15.9501 & 48.8837 & 0.00 & 1.3252 & 15.3040 & 48.0679 \\
\hline & 10.00 & 3.2957 & 20.0847 & 54.8263 & 10.00 & 1.9181 & 20.2319 & 55.7151 \\
\hline & 100.00 & 3.8452 & 22.3552 & 58.8541 & 100.00 & 2.0034 & 21.6126 & 58.1085 \\
\hline & 1000.00 & 3.9144 & 22.7031 & 59.2495 & 1000.00 & 2.0134 & 21.7898 & 58.3293 \\
\hline \multirow{4}{*}{2.00} & 0.00 & - & 15.1996 & 47.9949 & 0.00 & 1.1158 & 14.6152 & 47.1955 \\
\hline & 10.00 & 3.0449 & 19.4624 & 54.0107 & 10.00 & - & 19.3856 & 54.7637 \\
\hline & 100.00 & 3.6194 & 21.7474 & 58.0299 & 100.00 & - & 20.7108 & 57.1449 \\
\hline & 1000.00 & 3.6896 & 22.0932 & 58.4198 & 1000.00 & - & 20.8817 & 57.3706 \\
\hline
\end{tabular}


The natural frequencies are obtained by assuming $\mathrm{m}=0.01$ and $\mathrm{m}=0.1$ to find out the effect of varied damping coefficients on pipes dynamic behaviors .According to Fig. 1, it is verified that the values of natural frequencies decrease as $\mathrm{m}$ increases for all cases. For Case I and II, variation of damping coefficient has no visible effect on the natural frequencies and critical velocities. However, lower values of critical velocities are obtained with increasing damping coefficient in Case III. According to analysis results one observes much change in critical velocity in Case IV. At that case, the ratio between two natural frequencies (i.e the value of natural frequency when $\mathrm{m}=0.01 /$ the value of natural frequency when $\mathrm{m}=0.1$ ) is $\approx 66.56 \%$. Due to the pipe end conditions, importance of the damping coefficient greatly changes.
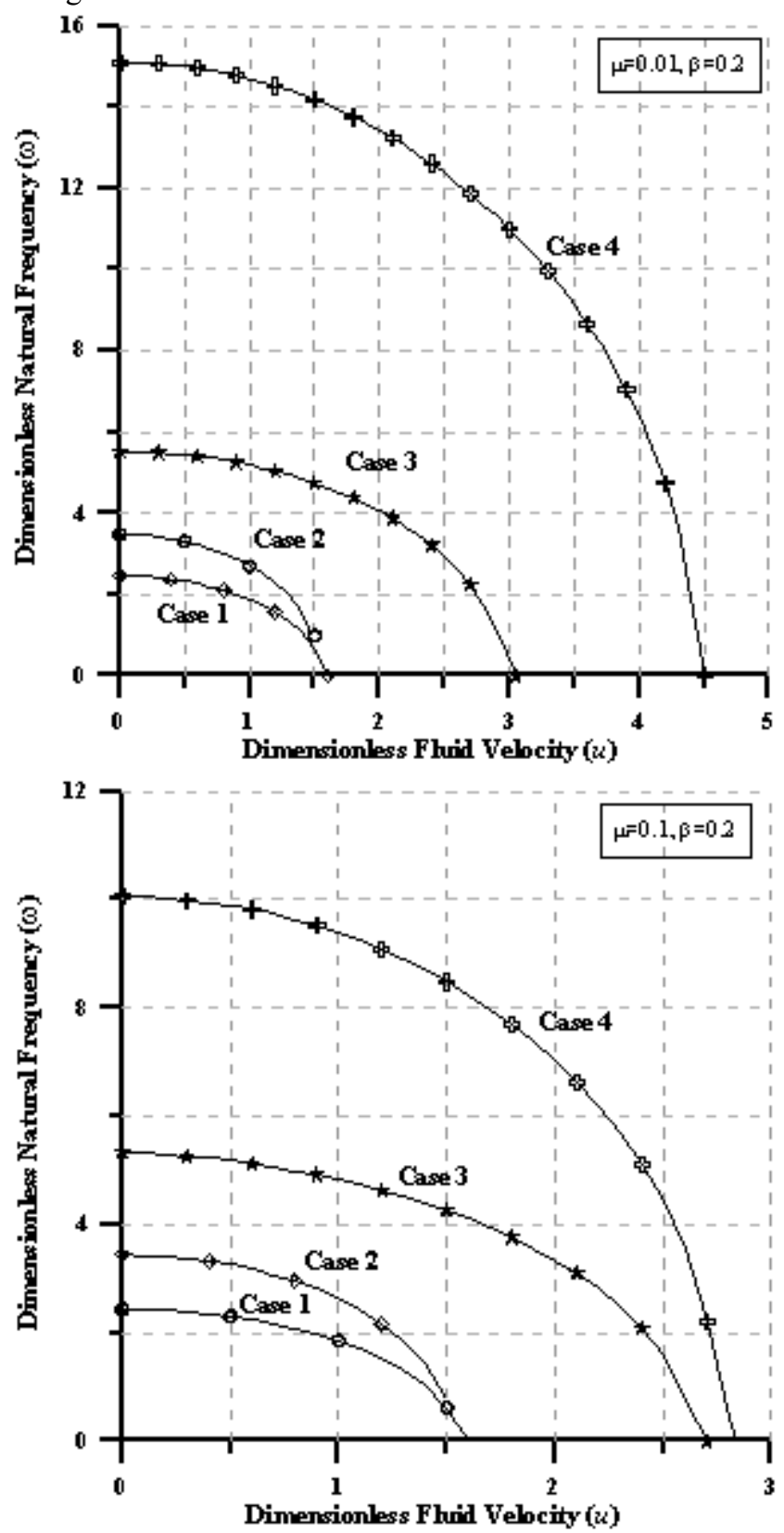

Figure 1. The natural frequency value versus fluid velocity for different mass ratios and damping coefficients

\section{Conclusions}

The dynamic behavior of a pipe conveying incompressible fluid supported by two different boundary conditions has been investigated in this study. The equations of motion of the pipe were obtained with a new approach with the assumptions of ideal fluid, which moves in the vertical direction with pipe and the pipe makes small harmonic oscillations. Differential equations obtained by applying the elastic clamped end, pinned end with torsional spring sliding end with torsional spring conditions on solutions have been solved by using software program.

The correlation between the values of natural frequencies and stiffnesses has been presented as tables. According to the results the values of natural frequencies are higher under tensile effects due to the immovable end conditions. For the analysis, five different values fluid velocity were assumed to confirm the effect of fluid velocity on the natural frequencies. As shown in results, this study has proved that the frequency values decrease with increasing fluid velocity.

In addition, the study developed with analysis of special values of stiffnesses of translational and rotational springs. The critical fluid velocities for the system instability are obtained by assuming $\mathrm{m}=0.01$ and $\mathrm{m}=0.1$. The values of critical velocity decrease with increasing damping coefficient. The rate of decrease of the natural frequencies and critical velocities due to the effect of damping coefficent has a small value for less rigid pipe systems.

\section{REFERENCES}

[1] Aitken, J., 1878. An account of some experiments on rigidity produced by centrifugal force. Philosophical Magazine, Series V 5, 81 - 105.

[2] Aldraihem, O.J., 2007. Analysis of the dynamic stability of collar- stiffened pipes conveying fluid. Journal of Sound and Vibraiton, 300, 453-465.

[3] Benjamın, T.B., 1961. Dynamics of a system of articulated pipes conveying fluid. I. Theory. Proceedings of the Royal Society (London) A 261, 457-486.

[4] Bourrieres, F.-J., 1939. Sur un phknomhe d'oscillation auto-entretenue en mkcanique des fluides rkels. Publications Scientijques et Techniques du Ministkre de l'Air, No. 147.

[5] Chang J.-R., Lin W.-J., Huang C.-J., Choi S.-T., 2010. Vibration and stability of an axially moving Rayleigh beam, Applied Mathematical Modelling 34 ,1482-1497.

[6] Chen S.S., 1972. Vibration and stability of a uniformly curved tube conveying fluid. Journal of Acoustical Society of America 51 223-232.

[7] Chen S.S., 1973. Out-of-plane vibration and stability of curved tubes conveying fluid. Journal of Applied Mechanics, $40,362-368$.

[8] Ghayesh M.H., Amabili M., Paidoussis M.P., 2012. ThermoMechanical Phase-Shift Determination im Coriolis 
mass-flowmeters with added masses, Journal of Fluids and Structures, 34, 1-13.

[9] Ergüt A., Dağlı B. Y., Sınır B. G., Dynamic analyze of pipe modeled by Rayleigh Theory conveying fluid. XVII. National Mechanic Conference, 5-9 September 2011, Elazı̆g (in Turkish).

[10] Gökkuş U., Eren A., Sınır B. G., 2009. Nonlinear dynamic analyze of current induced regular wave around the suspended marine pipeline with visco-elastic supports. TUBITTAK(Scientific and Technological Research Council of Turkey) 1001 (Project No:106M427)

[11] Han S. M., Benaroya H. And Wei T., March 1999. Dynamics Of Transversely Vibrating Beams Using Engineering Theories 225(5), 935-988.

[12] Koo G.H.\& Park Y.S., 1998. Vibration Reduction by Using Periodic Supports in a Piping System, Journal of Sound and Vibration 210(1), 53-68.

[13] Kang M. G., 1999. The Influence of Rotary Inertia of Concentrated Masses on the Natural Vibrations of a Clamped-Supported Pipe Conveying Fluid, Nuclear Engineering and Design, 196 281-292.

[14] Modarres-Sadeghi Y., Païdoussis M.P., Semler C. 2005. A Nonlinear Model for an Extensible Slender Flexible Cylinder Subjected to Axial Flow, Journal of Fluids and Structures.

[15] Modarres-Sadeghi Y., Païdoussis M.P., Semler C. 2008. Three Dimensional Oscillations of a Cantilever Pipe Conveying Fluid, International Journal of Non-Linear Mechanics 43 (1), 18-25.
[16] Ozkaya E., Pakdemirli M., Oz H. R., 1996. Non-Linear Vibration of a Beam-Mass System Under Different Boundary Conditions, Journal of Sound and Vibration, 199 (4), 679-696.

[17] Païdoussis M.P., Misra A.K., Van K.S., 1988. On the dynamics o f curved pipes transporting fluid. Part I: inextensible theory. Journal of Fluids and Structures, $2221-$ 244.

[18] Païdoussis, M.P., Issid, N.T. 1976, Experiments on parametric resonance of pipes containing pulsatile flow. Journal of Applied Mechanics, 43, 198-202.

[19] Païdoussis M.P., Luu T.P., Laithier B.E., 1986. Dynamics of Finite-Length Tubular Beams Conveying Fluid, Journal of Sound and Vibration 106(2), 311-331.

[20] Paidoussis M. P., 1998. Fluid-Structure Interactions Vol.1, first ed. Academis Press, London.

[21] Paidoussis M. P., 2003. Fluid-Structure Interactions Vol.2, first ed. Academis Press, London.

[22] Raszillier H., Alleborn N., Durst F., 1994. Effect Of A Concentrated Mass On Coriolis Flowmetering, Achive of Applied Mechanics, 64 (6), 373-382.

[23] Semercigil S.E, Turan O.F., Lu S. 1997. Employing Fluid Flow In A Cantilever Pipe for Vibration Control, Journal of Sound and Vibration, 205, 103-111

[24] Xua Y., Zhou D., 2009. Elasticity Solution Of Multi-Span Beams With Variable Thickness Under Static Loads, Applied Mathematical Modelling 33(7), 2951-2966. 